\title{
EDITORIAL Optimizing parental involvement in caring for preterm infants
}

Journal of Perinatology (2015) 35, 669-670; doi:10.1038/jp.2015.60

There is increased recognition that family-centered practices and parenting interventions are essential for promoting infant feeding, positioning, regulatory behaviors and social interactions after preterm birth. It is in this respect that the randomized clinical trial (RCT) by White-Trout et al. ${ }^{1}$ entitled 'Influences of H-HOPE intervention for premature infants on growth, feeding progression, and length of stay during initial hospitalization' offers important lessons in the current era of neonatal intensive care. $\mathrm{H}-\mathrm{HOPE}$ is an acronym for hospital to home transition: optimizing premature infants' environments. The H-HOPE program is built on a series of protocolized interventions by neonatal nurses, developmental psychologists and neonatologists in partnership with parents to address two major challenges in caring for preterm infants. The first is teaching caregivers to understand and respond to infant's cues that promote organized and coordinated suck, swallow and breathing during feeding. ${ }^{2}$ The second is to provide parents with management strategies for implementing developmentally informed practices during daily routines. ${ }^{3}$ The essential components of these multisensory interventions involve recognizing infant states and using touch, voice, positioning and social interactions before feedings. The key question is: 'Can these interventions promote parental involvement and concurrently improve infant growth, development and readiness for discharge?' An additional question is: 'Can these skills be implemented by mothers who have often been difficult to engage because of family stressors of poverty, minority status, limited maternal education and neighborhood distress?' It is to address these challenges that White-Traut et al. conducted a RCT comparing the H-HOPE intervention with a traditional parent-education program (PEP).

Who was studied? The cohort involved 182 infants born between 29- and 34-weeks gestation who were clinically stable and recruited from two central city neonatal intensive care nurseries. The mean infant gestation was 32.6 weeks (s.d. $=1.5$ ) and the mean birth weight was $1825 \mathrm{~g}$ (s.d. = 357) with $85 \%$ singleton, $52 \%$ female, 51\% Latina, 49\% African American and 30\% small for gestational age. To be recruited, these minority women had two or more social-environmental risks: less than a high school education, a family income less than $150 \%$ of the federal poverty level, a history of current mental illness or depression, residence in a neighborhood of concentrated disadvantage and household composition of more than one child under 2 years of age or four or more children under 4 years of age. Children were excluded if they had congenital anomalies, parenchymal brain injury, necrotizing enterocolitis, chronic lung disease, received oxygen via nasal cannula or had prenatal illicit drug exposures or HIV. Thus, the study design helped insure that the intervention would not be confounded by infants at highest risk for growth, feeding and developmental difficulties.

What was the structured intervention? Mothers gave the $\mathrm{H}-\mathrm{HOPE}$ intervention twice per day to their infants before feeding. The intervention consisted of $10 \mathrm{~min}$ of auditory (voice), tactile (message) and visual (eye-eye) stimulation followed by $5 \mathrm{~min}$ of vestibular (rocking) intervention. This combined intervention allows the parent to recognize the infant's cues as well as to calm the child during periods of distress. The mothers in the PEP comparison group received standard feeding and nursing attention and a series of parent educational handouts about infant care, holding the baby, bathing, sleep positions and sleep habits, safety of infant equipment and car safety. The majority (56-65\%) of women in both groups provided breast milk, with the interventions beginning at 9 days of age and when the infant was at 34 weeks post-menstrual age.

What was found? There was high fidelity in mothers learning the H-HOPE intervention. When the nurse advocate directly observed the mothers, there was $>90 \%$ adherence to the intervention checklists. Infants in the H-HOPE group grew significantly more rapidly than the PEP controls and demonstrated a $69-\mathrm{g}$ advantage in weight by day 28 and a $1.7-\mathrm{cm}$ advantage in length by day 20 . An indicator of brain growth, head circumference, was consistently higher throughout the study for the H-HOPE intervention group compared with the PEP controls. The $\mathrm{H}$-HOPE intervention did not reduce the time that infants required supplemental tube feedings or reduce duration of hospitalization compared with PEP controls.

What does this mean? First, in a most vulnerable population of minority women, characterized by two or more social-environmental risk factors, $97 \%$ agreed to regularly participate in their infant's care beginning in the second week of life. The second important finding is that mothers were able to learn to read and interpret their infants' behavioral cues, and thus more successfully help their infants learn feeding skills that promoted weight gain and linear growth. Third, this study offers valuable take-home lessons for improving partnerships with vulnerable families by providing evidence-based hands on learning opportunities.

What are these take-home lessons? First, there are many opportunities to start active parenting involvement in the care of their infants for this group of infants, who represent on a population basis a large number of infants admitted for neonatal intensive care, specifically those of 29 to 34 weeks gestational age. Second, additional studies will need to take place that also look at the impact of H-HOPE interventions on children with bronchopulmonary dysplasia, necrotizing enterocolitis, late-onset sepsis, retinopathy of prematurity, congenital malformations requiring surgery and parenchymal brain injury. The H-HOPE intervention adds to the growing body of research demonstrating improved weight gain and growth in hospitalized preterm infants who receive massage. ${ }^{4,5}$ However, it should be noted that there are gaps in measuring the long-term developmental impacts of these interventions on child and caregiver well-being. In particular, as social interactions are the prerequisite to communicative skills, social-emotional learning and adaptive behaviors, long-term studies assessing the impact on child health, development and parent-child interactions are needed. As there is increasing evidence that procedural stressors impact on vulnerable infants health and development, explicit interventions to optimize feeding skills and neural organization are critically needed for helping babies thrive in their hospital and home extra-uterine environments. $^{6}$ In this way, our postnatal neuroprotection practices that support and engage families can be linked to biomarkers, neurometrics and interventions that underlie child resiliency and enhance parental physical, behavioral and social well-being. 


\section{CONFLICT OF INTEREST}

The author declares no conflict of interest.

\section{ACKNOWLEDGEMENTS}

ME Msall's efforts are supported in part by grant T73MC11047-01-00 HRSADepartment of Health and Human Services. Leadership Education in Neurodevelop mental and Related Disorders Training Program (LEND). All the opinions expressed in this article are his own.

ME Msall

Section of Developmental and Behavioral Pediatrics, Kennedy Research Center on Intellectual and Developmental Disabilities, University of Chicago Medicine Comer Children's Hospital,

Chicago, IL, USA

E-mail: mmsall@peds.bsd.uchicago.edu

\section{REFERENCES}

1 White-Traut RC, Rankin RM, Yoder JC, Liu L, Vasa R, Geraldo V et al. Influence of $\mathrm{H}$-HOPE intervention for premature infants on growth, feeding, progression and length of stay during initial hospitalization. J Perinatol 2015; 35: 636-641.

2 Jiang S, Warre R, Qui X, O'Brien K, Lee K. Parents as practitioners in preterm care. Early Hum Dev 2014; 90: 781-785.

3 Evans T, Whittingham K, Sanders M, Colditz P, Boyd RN. Are parenting interventions effective in improving the relationship between mothers and their preterm infants? Infant Behav Dev 2014; 37: 131-154.

4 Vickers A, Ohlsson A, Lacy J, Horsley A. Massage for promoting growth and development of preterm and/or low birth-weight infants. Cochrane Database Syst Rev 2004; (2) Art. No.: CD000390.

5 Wang L, He JL, Zhang XH. The Efficacy of massage on preterm infants: a meta-analysis. Am J Perinatol 2013; 30: 731-738.

6 Msall ME. Physiological stress and brain vulnerability: understanding the neurobiology of connectivity in preterm infants. Ann Neurol 2011; 70(4): 523-524. 